\title{
PREVALÊNCIA DE EXCESSO DE PESO E FATORES DEMOGRÁFICOS E ECONÔMICOS ASSOCIADOS EM ADOLESCENTES DE MANAUS, A MAIOR CIDADE DO NORTE DO BRASIL
}

\author{
PREVALENCE OF OVERWEIGHT AND ASSOCIATED DEMOGRAPHIC AND ECONOMIC FACTORS \\ AMONG ADOLESCENTS OF MANAUS, THE LARGEST CITY OF NORTHERN BRAZIL
}

\section{André de Araújo Pinto ${ }^{\mathrm{a}^{*}}$, Rita Maria dos Santos Puga Barbosa ${ }^{\mathrm{b}^{* *}}$, Markus Vinicius Nahas ${ }^{\mathrm{c}^{* * *}}$, Andreia Pelegrini $\mathrm{d}^{*}$}

\author{
aandrefsaude@hotmail.com, brita.puga@outlook.com, cmarkusvnahas@gmail.com, dandreia.pelegrini@udesc.br \\ *Universidade do Estado de Santa Catarina - Florianópolis (SC), Brasil \\ **Universidade Federal do Amazonas - Manaus (AM), Brasil \\ ***Universidade Federal de Santa Catarina - Florianópolis (SC), Brasil
}

\section{RESUMO}

Introduçáo: Estimativas da prevalência de excesso de peso em adolescentes brasileiros foram geradas em diversos estados, mas sobre os adolescentes da região Norte a carência de dados é evidente. Objetivo: Estimar a prevalência de excesso de peso e sua associação com os fatores demográficos e econômicos em adolescentes de Manaus (AM). Metodologia: Estudo transversal conduzido com 1.107 adolescentes (627 moças), com idades de 14 a 19 anos, de Manaus, realizado no ano de 2011. $\mathrm{O}$ excesso de peso foi classificado utilizando-se o índice de massa corporal e adotando-se os pontos de corte para sexo e idade recomendados pela International Obesity Task Force (IOFT). As variáveis independentes foram sexo, faixa etária, renda familiar e nível de escolaridade da mãe. As associaçóes foram testadas por meio da regressáo de Poisson. Resultados: A prevalência de excesso de peso foi de $37,4 \%$ (IC95\% = 34,6-39,8). Os adolescentes do sexo masculino (RP = 2,54; IC95\% = 2,12-3,03), os de 14 a 16 anos de idade $(\mathrm{RP}=1,23$; IC95\% = 1,06-1,44) e os filhos de mãe com maior nível de escolaridade $(\mathrm{RP}=1,66$; IC95\% $=1,44-1,92)$ apresentaram maior probabilidade de ter excesso de peso quando comparados às do sexo feminino, aos com idade entre 18 e 19 anos e aos filhos de mães com menor nível de escolaridade, respectivamente. Conclusáo: A prevalência de excesso de peso foi elevada em relação a outros estudos brasileiros. Medidas preventivas para o excesso de peso devem ser direcionadas principalmente aos adolescentes do sexo masculino, aos mais jovens e aos filhos de mães com maior nível de escolaridade.

Palavras-chave: Estado nutricional; saúde do adolescente; antropometria; epidemiologia.

\section{ABSTRACT}

Introduction: Estimates of overweight prevalence among Brazilian adolescents have been made in several states, but the scarcity of data about the adolescents from Northern region is evident. Objective: To estimate the prevalence of excess weight and its association with demographic and economic factors in adolescents from Manaus, Amazonas, Brazil. Methodology: A cross-sectional study was conducted among 1,107 adolescents (627 girls), with ages ranging from 14 to 19 years, in Manaus city, in the year 2011. Overweight was classified by using body mass index and adopting the cut-off points for gender and age recommended by the International Obesity Task Force (IOTF). The independent variables were gender, age, household income and educational level of the mother. Associations were tested using Poisson regression. Results: The prevalence of overweight was $37.4 \%$ (95\% $\mathrm{CI}=34.6-39.8)$. The adolescents who are male $(\mathrm{PR}=2.54 ; 95 \% \mathrm{CI}=2.12-3.03)$, the ones who are 14 to 16 years old $(\mathrm{PR}=1.23 ; 95 \% \mathrm{CI}=1.06-1.44)$, and the children of mothers with higher level of education $(\mathrm{PR}=1.66 ; 95 \% \mathrm{CI}=1.44-1.92)$ were more likely to have excess weight when compared 
to the female ones, to those aged 18 to 19 years and to children of mothers with lower schooling level, respectively. Conclusion: The overweight prevalence was high in relation to other Brazilian studies. The preventive measures for excess weight should be directed mainly to male adolescents, to the youngers and to children of mothers with higher education level.

Keywords: Nutritional status; adolescent health; anthropometry, epidemiology.

\section{Introdução}

Atualmente, o cenário epidemiológico mundial tem destacado a necessidade do combate à obesidade, pois seus efeitos deletérios sobre a saúde da população estão cada vez mais em evidência ${ }^{1}$. A modernização da sociedade, especialmente por meio dos avanços tecnológicos, tem contribuído para que as pessoas incorporem hábitos mais nocivos à saúde ${ }^{2,3}$, optando por não realizar grandes esforços, o que consequentemente aumenta o risco do desenvolvimento de doenças como hipercolesterolemia, hipertrigliceridemia, hipertensão arterial e diabetes em todas as fases da vida ${ }^{4}$.

No Brasil, assim como em outros países de economia crescente, tem ocorrido nos últimos anos um fenômeno conhecido como transiçáo nutricional, caracterizado pela redução da pobreza nas camadas menos favorecidas, enquanto os casos de excesso de peso (sobrepeso e obesidade) ascendem alarmantemente 5 . De acordo com o Instituto Brasileiro de Geografia Estatística (IBGE), a prevalência do excesso de peso em adultos sofreu um aumento de mais de $100 \%$ nos anos de 1974 a 1985 $(28,9 \%)$ e de 2008 a $2009(63,8 \%)^{6}$. Os dados alusivos aos adolescentes são ainda mais estarrecedores, pois nos mesmos períodos a prevalência de excesso de peso mais que quadriplicou (eram de $6,2 \%$ e $25 \%$, respectivamente), reforçando o cenário preocupante da atualidade, o qual exige controle imprescindível do peso corporal.

Em 2007, em revisão sistemática realizada por Araújo et al. ${ }^{7}$, observou-se um número expressivo de estudos epidemiológicos com foco no excesso de peso em adolescentes no Brasil, cuja prevalência oscilou de 4\% a 31\%. Em 2014, Hobold e Arruda ${ }^{8}$ sustentaram os achados de Araújo et al. ${ }^{7}$; no entanto, a prevalência de excesso de peso encontrada variou de $7,4 \%$ a $36,5 \%$. Curiosamente, em ambas as revisóes, houve uma maior concentração de dados das regióes Sul e Sudeste, e poucos estudos com dados das regióes Nordeste e CentroOeste foram rastreados ${ }^{7,8}$. Na regiáo Norte, apenas um estudo conduzido com adolescentes acreanos em 2014 foi rastreado ${ }^{8}$, o que significa escassez de informaçôes sobre as prevalências de excesso de peso na região.

Além do interesse pelos dados de prevalência, pesquisadores das áreas de atividade física e saúde têm focado, também, na identificação dos fatores de maior exposição a essa condiçãa ${ }^{3,7-12}$. Evidências demonstraram que os adolescentes do sexo masculino ${ }^{7,8}$, os mais jovens $s^{6,13,14}$, os de famílias com melhor aquisição financei$\mathrm{ra}^{3,8,12}$ e os filhos de mães com maior nível de escolarida$\mathrm{de}^{10,15}$ estão mais expostos ao excesso de peso. Todavia, tais evidências fazem parte, em sua maioria, de estudos realizados com adolescentes das regiōes Sul e Sudeste ${ }^{7,8}$, sendo possível que tais fatores divirjam conforme a região estudada - principalmente no Brasil, um país de dimensões continentais e multiculturais.

Diante desse cenário, em que o excesso de peso tem sido considerado um problema de saúde pública emergente $^{16}$, fica claro que o panorama do Brasil deve-se, principalmente, a contribuiçóes empíricas realizadas em regiốes economicamente mais favorecidas, sendo que nas regióes em crescente desenvolvimento, como a regiáo Norte, na qual se situam grandes centros, ainda é desconhecido o real panorama do excesso de peso em adolescentes. Isso dificulta o desenvolvimento de políticas sociais destinadas à prevenção, ao tratamento e ao controle da epidemia de obesidade no país, as quais podem não estar direcionadas a todas as regióes. Diante disso, o objetivo deste estudo foi estimar a prevalência de excesso de peso e sua associação com os fatores demográficos e econômicos em adolescentes de Manaus (AM).

\section{Metodologia}

Trata-se de um estudo transversal epidemiológico, de base escolar, vinculado ao macroprojeto "Estilo de vida e saúde de adolescentes do ensino médio no Amazonas". O projeto de pesquisa foi aprovado pelo Comitê de Ética e Pesquisa em seres humanos da Universidade Federal do Amazonas (sob o registro no CAAE 0302.0.115.00011). Este estudo limitou-se a investigar os adolescentes matriculados nas escolas públicas da rede estadual de educaçâoo da cidade de Manaus.

A capital do Amazonas está situada na regiáo Norte do país, possui área de $11.401,09 \mathrm{~km}^{2}$ e 63 bairros distribuídos em seis zonas geográficas. Com população de 1.802.014 habitantes, a mais populosa da região Norte, a cidade é também considerada a sétima mais populosa do país, e seu Índice de Desenvolvimento Humano Municipal (IDHM) é de $0,737^{17}$. Manaus apresenta uma taxa de $51,23 \%$ de jovens (15-17 anos) com ensino fundamental completo, renda per capita de $\mathrm{R} \$ 790,27$ 
e índice Gini 0,61 (que retrata baixa desigualdade de renda entre seus habitantes), sendo seu percentual de extrema pobreza $3,75 \%{ }^{17}$.

Segundo as informaçóes da Secretaria de Estado de Educação, 78.498 adolescentes, distribuídos em 102 escolas estavam matriculados no ano de 2011. A amostra foi determinada segundo procedimentos prévios de amostragem $^{18}$, a partir de uma população finita, considerando-se: prevalência estimada de 50\% (desfecho desconhecido), nível de significância de 1,96 (intervalo de confiança de $95 \%$ ), erro tolerável de $4 \%$ e efeito de delineamento de 1,5 . Com o intuito de minimizar eventuais perdas e recusas, acrescentou-se mais $20 \%$ à amostra final, estimando-se uma amostra necessária de 1.072 adolescentes.

A seleção da amostra foi determinada em três estágios: (i) proporcional por zonas geográficas; (ii) estratificado por escolas; e (iii) conglomerados de turmas, levando-se em consideração turno, série e turmas. Para este estudo, todas as escolas foram consideradas elegíveis e inicialmente foram classificadas quanto ao número total de alunos (pequeno porte para até 200 alunos; médio porte para de 200 a 499 alunos e grande porte para 500 alunos ou mais). Posteriormente, foi realizado o sorteio das escolas de acordo com o porte em cada regiáo geográfica. Na sequência foi considerado o turno de estudo e o ano escolar, e os todos os adolescentes das turmas sorteadas foram convidados para participar do estudo. Esse procedimento elevou o tamanho da amostra para 1.413 adolescentes, dos quais 192 foram excluídos por ausência de dados válidos.

Todo o processo de coleta foi realizado nas dependências das próprias escolas, em dias e horários acordados previamente com os responsáveis e professores das instituiçôes. Os adolescentes foram informados antecipadamente sobre os objetivos da pesquisa e convidados a fazer parte dela. Foram incluídos neste estudo os adolescentes que aceitaram participar voluntariamente, com idade de 14 a 19 anos, e que estavam presentes em sala de aula no dia da coleta de dados. Todos eles assinaram o Termo de Assentimento e entregaram o Termo de Consentimento Livre e Esclarecido assinado por seus responsáveis legais (para aqueles com idade inferior a 18 anos) ou por eles mesmos (idade superior a 18 anos).

Todas as variáveis do estudo foram coletadas mediante aplicação de questionário autoadministrado, contendo informaçóes demográficas, econômicas, de massa corporal e de estatura, as quais foram autorreferidas pelos adolescentes. A variável dependente deste estudo, o índice de massa corporal $(\mathrm{IMC}=$ peso corporal $[\mathrm{kg}] /$ estatura $\left.{ }^{2}\left[\mathrm{~m}^{2}\right]\right)$, foi determinado a partir das medidas autorreferidas (peso corporal e estatura) e posteriormente classificado (baixo peso, eutrófico, sobrepeso e obesidade) de acordo com os pontos de corte para sexo e idade sugeridos pela International Obesity Task Force (IOTF) ${ }^{19}$. Estudos epidemiológicos realizados no Brasil $^{20,21}$ e no mundo ${ }^{22,23}$ têm utilizado com frequência medidas de massa corporal e estatura autorreferidas para determinar o estado nutricional de adolescentes. Além disso, optou-se por esse procedimento devido à ausência de fomento para a realização da pesquisa e de uma equipe com total disponibilidade para executar a coleta de dados. Foram considerados com excesso de peso os adolescentes que apresentaram sobrepeso e/ou obesidade 5 .

Foram coletadas informaçóes referentes ao sexo (masculino e feminino), à faixa etária (de 14 a 15 anos, de 16 a 17 anos e de 18 a 19 anos), ao ano de estudo (primeiro, segundo e terceiro ano) e ao turno (matutino, vespertino e noturno). As variáveis econômicas foram a renda familiar mensal e o nível de escolaridade da mãe. Esta primeira foi determinada a partir do salário mínimo vigente de 2011 ( $\mathrm{R} \$ 545,00)$ e levou em consideração o somatório da renda de todos os trabalhadores presentes na residência, por meio da seguinte questáo: "qual a renda mensal da sua família?". As opçóes para resposta foram: a) até dois salários; b) de três a cinco salários; c) de seis a 10 salários; e d) 11 salários ou mais. Em virtude da baixa frequência de respostas "c" $(6,1 \%)$ e "d" (2,3\%), optou-se por reagrupá-las em três categorias: (i) até dois salários; (ii) de três a cinco salários; e (iii) seis salários ou mais. Para identificar o nível de escolaridade da mãe os estudantes responderam à seguinte pergunta: "qual a alternativa melhor indica o nível de escolaridade da sua mãe?”. As alternativas para as possíveis respostas foram: a) nunca estudou; b) estudou até o ensino fundamental; c) estudou até o ensino médio; d) estudou até a faculdade; e) não sei. Para fins de análise, o nível de escolaridade materno foi dicotomizado em: (i) até oito anos de estudo (não estudou $[7,4 \%]+$ ensino fundamental $[44,3 \%]$ ); e (ii) mais de oito anos (ensino médio [26,2\%] + até a faculdade [13,2\%]). Os adolescentes que responderam com a alternativa "não sei” $(8,8 \%)$, não foram considerados na dicotomização.

As análises de dados foram administradas no software IBM SPSS Statistics, versão 20.0, com nível de significância estabelecido de 5\%. Utilizou-se a estatística descritiva, por meio de frequências (absoluta e relativa), e inferencial. $\mathrm{O}$ teste qui-quadrado foi empregado com a intençáo de identificar possíveis interaçôes entre as variáveis. A regressão de Poisson, com estimativa robusta, bruta e ajustada por todas as varáveis, foi utilizada para averiguar as associaçóes entre a variável desfecho (excesso de peso) e as variáveis independentes (sexo, faixa etária, renda familiar e escolaridade da mãe), estimando-se a razão de prevalência $(\mathrm{RP})$ e os intervalos de confiança de $95 \%$ (IC95\%). 


\section{Resultados}

Foram coletadas informaçóes de 1.221 estudantes, dos quais 114 foram desconsiderados para a análise por não se enquadrarem na faixa etária estabelecida, resultando em uma amostra final de 1.107 adolescentes (627 moças). A maioria dos adolescentes possuía idade entre 16 e 17 anos $(57,2 \%)$, cursava o primeiro ano do ensino médio $(39,8 \%)$, estudava no turno vespertino $(54,2 \%)$, apresentava renda familiar de até dois salários $(57,0 \%)$ e mães tinham até oito anos de estudo (56,8\%). Em relação ao estado nutricional, constatou-se que a maior parte deles apresentou peso normal $(55,7 \%)$ (Tabela 1$)$.

Tabela 1: Características gerais dos adolescentes do ensino médio da rede pública estadual de Manaus (AM), Brasil (2011).

\begin{tabular}{|c|c|c|c|c|}
\hline \multirow{2}{*}{ Variáveis } & \multirow{2}{*}{ Total n (\%) } & \multicolumn{2}{|c|}{ Sexo } & \multirow{2}{*}{ p-valor* } \\
\hline & & Masculino n (\%) & Feminino n (\%) & \\
\hline Faixa etária, em anos & & & & 0,062 \\
\hline $14-15$ & $221(19,1)$ & $94(44,5)$ & $117(55,5)$ & \\
\hline $16-17$ & $633(57,2)$ & $255(40,6)$ & $378(59,4)$ & \\
\hline $18-19$ & $263(23,8)$ & $131(49,0)$ & $132(51,0)$ & \\
\hline Ano de estudo & & & & 0,043 \\
\hline Primeiro ano & $438(39,8)$ & $207(47,3)$ & $231(52,7)$ & \\
\hline Segundo ano & $386(35,1)$ & $149(38,6)$ & $237(61,4)$ & \\
\hline Terceiro ano & $276(24,9)$ & $119(43,1)$ & $157(56,9)$ & \\
\hline Turno & & & & 0,044 \\
\hline Matutino & $294(27,5)$ & $134(45,6)$ & $160(54,4)$ & \\
\hline Vespertino & $580(54,2)$ & $234(40,3)$ & $346(59,7)$ & \\
\hline Noturno & $196(18,3)$ & $98(50,0)$ & $98(50,0)$ & \\
\hline Renda famíliar & & & & $<0,001$ \\
\hline Até 2 salários & $617(57,0)$ & $233(37,8)$ & $384(62,2)$ & \\
\hline De 3 a 5 salários & $374(34,6)$ & $179(47,9)$ & $196(52,1)$ & \\
\hline 6 salários ou mais & $91(8,4)$ & $58(63,7)$ & $33(36,3)$ & \\
\hline Escolaridade materna & & & & $<0,001$ \\
\hline Até 8 anos & $571(56,8)$ & $232(40,6)$ & $339(59,4)$ & \\
\hline Mais de 8 anos & $435(43,2)$ & $245(56,3)$ & $190(43,7)$ & \\
\hline Status do peso & & & & $<0,000$ \\
\hline Baixo peso & $76(6,9)$ & - & $76(100,0)$ & \\
\hline Eutróficos & $617(55,7)$ & $184(29,8)$ & $433(70,2)$ & \\
\hline Sobrepeso & $323(29,2)$ & $227(70,3)$ & $96(29,7)$ & \\
\hline Obesidade & $91(8,2)$ & $69(75,8)$ & $22(24,2)$ & \\
\hline
\end{tabular}

n: frequência absoluta; \%: frequência relativa; *teste qui-quadrado.

Fonte: Elaborada pelos autores a partir dos dados coletados, 2017.

De acordo com a Tabela 2, a prevalência de excesso de peso (sobrepeso/obesidade) entre os adolescentes foi de $37,4 \%$. As maiores proporçôes do desfecho foram observadas no sexo masculino $(61,7 \%)$, nos adolescentes com idades entre 14 e 15 anos (45,0\%), nos com renda familiar de seis salários ou mais $(40,7 \%)$ e nos filhos de mães com escolaridade de até oito anos de estudo $(55,9 \%)$. 
Tabela 2: Prevalência de excesso de peso de acordo com as variáveis demográficas e econômicas em adolescentes de Manaus (AM), Brasil (2011).

\begin{tabular}{lll} 
Variáveis & \multicolumn{2}{l}{ Excesso de peso } \\
Sexo & P & IC*95\% \\
$\begin{array}{l}\text { Masculino } \\
\text { Feminino }\end{array}$ & 61,7 & $58,7-64,1$ \\
Faixa etária, em anos & 18,8 & $16,5-20,8$ \\
\hline 14-15 & 45,0 & $42,1-47,4$ \\
$16-17$ & 34,1 & $31,4-36,4$ \\
$18-19$ & 39,2 & $36,3-41,7$ \\
Renda familiar & & \\
Até 2 salários & 35,7 & $32,8-38,0$ \\
De 3 a 5 salários & 39,8 & $36,9-42,2$ \\
6 salários ou mais & 40,7 & $37,9-43,1$ \\
Escolaridade materna & & \\
Até 8 anos & 29,6 & $26,9-31,8$ \\
Mais de 8 anos & 55,9 & $52,9-58,4$ \\
Total & $\mathbf{3 7 , 4}$ & $\mathbf{3 4 , 6 - 3 9 , 8}$ \\
\hline
\end{tabular}

P: prevalência; *intervalo de confiança para proporção.

Fonte: Elaborada pelos autores a partir dos dados coletados, 2017.

$\mathrm{Na}$ Tabela 3 foram apresentados os resultados da análise de associação, considerando-se o excesso de peso como desfecho. $\mathrm{Na}$ análise bruta, todas as variáveis se associaram ao excesso de peso, exceto a renda familiar. Após o ajuste de todas as variáveis, observou-se maior probabilidade de que apresentassem o desfecho os adolescentes do sexo masculino $(\mathrm{RP}=2,54$; IC95\% $=2,12-3,03)$, quando comparados às do sexo feminino. Os adolescentes de 14 e 15 anos ( $\mathrm{RP}=1,23$; IC95\% $=1,06-1,44)$ e aqueles cujas mães tinham mais de oito anos de estudo ( $\mathrm{PR}=1,66$; IC95\% $=1,44-1,92)$ apresentaram maior probabilidade de ter excesso de peso em relação aos seus respectivos pares de 16 e 17 anos e filhos de mães com menor grau de escolaridade.

Tabela 3: Razões de prevalência de excesso de peso e fatores associados em adolescentes de Manaus (AM), Brasil (2011).

\begin{tabular}{lll} 
Variáveis & $\begin{array}{l}\text { Análise bruta } \\
\text { RP (IC95\%) }\end{array}$ & $\begin{array}{l}\text { Análise ajustada* } \\
\text { RP (IC95\%) }\end{array}$ \\
\hline $\begin{array}{l}\text { Sexo } \\
\text { Masculino }\end{array}$ & $3,28(2,74-3,91)$ & $2,54(2,12-3,03)$ \\
Feminino & 1 & 1 \\
\hline Faixa etária, em anos & & \\
$14-15$ & $1,32(1,10-1,59)$ & $1,23(1,06-1,44)$ \\
$18-19$ & $1,15(0,95-1,38)$ & $1,10(0,93-1,29)$ \\
$16-17$ & 1 & 1 \\
\hline
\end{tabular}

continua...
Tabela 1: Continuação.

\begin{tabular}{lll}
\hline Variáveis & $\begin{array}{l}\text { Análise bruta } \\
\text { RP (IC95\%) }\end{array}$ & $\begin{array}{l}\text { Análise ajustada* } \\
\text { RP (IC95\%) }\end{array}$ \\
\hline Renda familiar & & \\
\hline 6 salários ou mais & $1,14(0,87-1,49)$ & $0,82(0,65-1,05)$ \\
De 3 a 5 salários & $1,11(0,95-1,32)$ & $1,06(0,89-1,18)$ \\
Até 2 salários & 1 & 1 \\
Escolaridade materna & & $\mathbf{1 , 6 6 ( 1 , 4 4 - 1 , 9 2 )}$ \\
Mais de 8 anos & $1,89(1,62-2,20)$ & 1 \\
\hline Até 8 anos & 1 & \\
\hline
\end{tabular}

*Ajustada por todas as variáveis.

Fonte: Elaborada pelos autores a partir dos dados coletados, 2017.

\section{Discussão}

O objetivo principal deste estudo foi estimar a prevalência de excesso de peso e sua associação com fatores demográficos e econômicos em adolescentes de Manaus. Elevada prevalência do desfecho $(37,4 \%)$ foi observada entre os adolescentes, sendo mais frequente nos adolescentes sexo masculino, nos mais jovens (de 14 a 15 anos) e nos filhos de máes com maior grau de escolaridade.

A prevalência de excesso de peso verificada nos adolescentes avaliados mostrou-se elevada e preocupante se comparada à de adolescentes de faixa etária semelhante em outros locais do país, como apontam estudos realizados em Recife (PE), com prevalência de $10,4 \%{ }^{11}$, Rio Branco (AC), com 29,5\% ${ }^{9}$, Montes Claros (MG), com $30,4 \%{ }^{10}$, e Apucarana (PR), com $31,3 \%{ }^{3}$. Prevalências tão elevadas quanto a encontrada neste estudo só foram observadas em adolescentes americanos, nos estados de Arkansas (37,5\%), Mississipi (44,5\%) e Geórgia (37,3\%), conforme mapeamento realizado nos Estados Unidos $^{24}$. Partindo disso, acredita-se que o problema do excesso de peso seja típico, mesmo em países e estados economicamente distintos, e especialmente em Manaus faz-se necessário a adoção de medidas urgentes para combatê-lo.

Tais diferenças entre as prevalências de excesso de peso, no entanto, podem ser explicadas pelas disparidades geográficas, em função, principalmente, de hábitos peculiares de determinadas culturas ${ }^{24}$ - na regiáo Norte, por exemplo, há um maior consumo de carnes, farinhas e massas, oriundo dos costumes típicos locais. Além disso, é provável que diferentes critérios metodológicos, e também os métodos estatísticos, muitas vezes empregados para classificar o estado nutricional, possam resultar em oscilaçóes entre as prevalências dos estudos. No que tange à elevada prevalência de excesso de 
peso observada, diversos fatores podem ter contribuído para essa ocorrência, destacando-se os comportamentos de restrição alimentar, a frequência e a intensidade de atividades físicas, o tempo gasto em comportamento sedentário, o estado nutricional pregresso e o estado nutricional dos pais ${ }^{1,24}$. No entanto essas variáveis precisam ser investigadas na população alvo desta pesquisa, uma vez que este estudo limitou-se apenas aos fatores demográficos e econômicos.

Os adolescentes do sexo masculino apresentaram maior probabilidade de ter excesso de peso em relação ao sexo oposto. Esse resultado vai ao encontro dos achados apontados em revisôes sistemáticas realizadas em $2007^{7}$ e, mais tarde, em $2014^{8}$, que observaram, na maioria das evidências, maior exposição do sexo masculino ao excesso de peso. Essa tendência foi observada na Pesquisa de Orçamentos Familiares (POF) realizada entre 2002 e $2003^{25}$ e entre 2008 e $2009^{6}$, mas náo na Pesquisa Nacional de Saúde do Escolar (Pense) de 2015, cujas prevalências de excesso de peso foram semelhantes em ambos os $\operatorname{sexos}^{26}$. Maiores prevalências de excesso de peso no sexo masculino foram constadas em revisão sistemática com dados de adolescentes de diversos países de todos os continentes ${ }^{27}$.

Embora pareça consenso que os adolescentes do sexo masculino sejam mais expostos ao excesso de peso do que seus pares opostos ${ }^{6-8,25}$, as explicaçóes para isso não estão bem esclarecidas, pois se por um lado a intensa atividade hormonal, típica da adolescência, aumenta as chances de que as moças acumulem mais gordura corporal do que os rapazes ${ }^{12}$, por outro, as adolescentes tendem a ser mais cuidadosas em relação à alimentação do que seus pares, principalmente aquelas consideradas eutróficas ${ }^{28}$. Além disso, considerando que as prevalências são geralmente calculadas pelo $\mathrm{IMC}^{27}$, é bem provável que para os adolescentes do sexo masculino tenha ocorrido uma superestimação dos valores brutos (por estes possuírem mais massa magra), pois essa distinção entre massa gorda e massa magra não é bem identificada pelo $\mathrm{IMC}^{29}$.

A faixa etária também se associou ao excesso de peso, sendo observada uma maior exposição dos adolescentes mais jovens (de 14 a 15 anos) ao desfecho. Resultados semelhantes foram observados em estudo conduzido com adolescentes de Fortaleza (CE) ${ }^{13}$, Capão da Canoa $(\mathrm{RS}){ }^{14} \mathrm{e}$ em dois levantamentos nacionais realizados no Brasi $^{6,25}$. Uma possível explicação para essa evidência pode estar relacionada à preocupação com o aspecto físico, especialmente em adolescentes com idades mais avançadas, que parecem estar mais atentos à aparência física ${ }^{30}$. Além disso, em Manaus é comum que os adolescentes, em especial aqueles do segundo e terceiro ano do ensino médio, realizem algum tipo de estágio extracurricular e, por permanecerem menos tempo em casa, estejam menos expostos aos excessos nos hábitos alimentares em suas residências, o que seria outra hipótese para explicar esse resultado.

Outro fator de risco para o excesso de peso foi o maior nível de escolaridade da mãe, o que também foi confirmado nos estudos realizados com adolescentes de Montes Claros $(\mathrm{MG})^{10}$ e de Pelotas (RS) $)^{15}$, mas não com os adolescentes de Florianópolis (SC $)^{12}$ e Pernambuco ${ }^{31}$. Essa comparaçáo entre os estudos deve ser feita com cautela, haja vista que o uso de diferentes categorizaçóes para classificar o nível de escolaridade da mãe não segue o mesmo padrão entre as pesquisas. Apesar de não se ter explicaçõos claras sobre essa associação em estudos prévios, presume-se que o nível de escolaridade da mãe possa interferir na condição econômica da família, facilitando comportamentos responsáveis pelo aumento do risco de desenvolvimento de excesso de peso, com destaque para a escolha de alimentos pouco nutritivos e indução de comportamento sedentário ${ }^{10}$. Isso de fato parece procedente, pois embora náo tenha sido observada uma associação estatisticamente significativa entre a renda familiar e o excesso de peso neste estudo, a maior proporção de adolescentes que apresentaram o desfecho era de famílias com renda superior a três salários mínimos.

Entre as limitações do estudo, pode-se destacar que o fato de ter sido conduzido com populaçáo de base escolar impossibilita a generalização dos resultados para os demais adolescentes; o delineamento transversal empregado não permite estabelecer qualquer relação de causa-efeito entre as variáveis estudadas, porém estudos com esse delineamento são importantes para descrever dados relacionados à saúde; as medidas autorreferidas de peso e estatura para estimar a prevalência de excesso de peso podem ter sido subestimadas ou superestimadas pelos adolescentes, e principalmente por essa razão a prevalência encontrada precisa ser vista com cautela. Apesar disso, este estudo apresenta pontos fortes, destacando-se que se trata do primeiro estudo empírico a respeito do tema abrangendo a maior cidade do Amazonas; a utilização de uma amostra representativa de adolescentes e com perda amostral inexistente; e que os dados apresentados podem ser úteis para fins de comparação com estudos futuros, especialmente por se tratar de um assunto com cada vez mais destaque na saúde pública.

\section{Conclusão}

A prevalência de excesso de peso encontrada nos adolescentes de Manaus foi considerada elevada se comparada com outros estudos realizados no Brasil. Os 
adolescentes do sexo masculino, os mais jovens e os filhos de mães com maior nível de escolaridade foram os subgrupos que apresentaram maior exposição aos riscos sucessivos do excesso de peso. Diante dos achados preocupantes, é de extrema urgência o desenvolvimento de estratégias que visem prevenir, controlar e tratar o excesso de peso corporal na população adolescente do município Manaus, considerando a relevância dos fatores demográficos e econômicos no desfecho apresentado.

\section{Referências}

1. Cardoso LO, Engstrom EM, Leite IC, Castro IRR. Socioeconomic, demographic, environmental and behavioral factors associated with overweight in adolescents: a systematic literature review. Rev Bras Epidemiol. 2009;12(3):378-403.

2. Ariza C, Ortega-Rodríguez E, Sánchez-Martínez F, Valmayor S, Juárez O, Pasarín MI. La prevención de la obesidad infantil desde una perspectiva comunitaria. Atención Primaria. 2015;47(4):246-55.

3. Guedes DP, Paula IG, Guedes JERP, Stanganelli LCR. Prevalência de sobrepeso e obesidade em crianças e adolescentes estimativas relacionadas ao sexo, à idade e à classe socioeconômica: Rev Bras Educ Fís Esp. 2006;20(3):151-63.

4. Kneipp C, Habitzreuter F, Mezadri T, Hofelmann DA. Excesso de peso e variáveis associadas em escolares de Itajaí, Santa Catarina, Brasil. Cien Saúde Colet. 2015;20(8):2411-22.

5. Coutinho W. Consenso latino-americano de obesidade. Arq Bras Endocrinol Metab. 1999;43(1):21-67.

6. Instituto Brasileiro de Geografia e Estatística. Pesquisa de Orçamentos Familiares: antropometria e estado nutricional de crianças, adolescentes e adultos do Brasil. Rio de Janeiro: IBGE; 2010.

7. Araújo VC, Konrad LM, Rabacow FM, Graup S, Amboni R, Farias Júnior JC. Prevalência de excesso de peso em adolescentes brasileiros: um estudo de revisão sistemática. Rev Bras Ativ Fis Saúde. 2012;12(3):79-87.

8. Hobold E, Arruda M. Prevalência de sobrepeso e obesidade de criança e adolescentes no Brasil: uma revisão sistemática. Arq Ciências Saúde. 2014;18(3):189-97.

9. Silva Júnior LM, Santos AP, Souza OF, Farias ES. Prevalência de excesso de peso e fatores associados em adolescentes de escolas privadas de regiáo urbana na Amazônia. Rev Paul Pediatr. 2012;30(2):217-22.

10. Guedes DP, Miranda Neto JT, Almeida MJ, Silva AJRM. Impacto de fatores sociodemográficos e comportamentais na prevalência de sobrepeso e obesidade de escolares. Rev Bras Cineantropom Desemp Hum. 2010;12(1):221-31.

11. Griz LH, Viégas MM, Barros M, Griz AL, Freese E, Bandeira F. Prevalência de obesidade central em grande amostra de adolescentes de escolas públicas em Recife, Brasil. Arq Bras Endocrinol Metab. 2010;54(7):607-11.

12. Pelegrini A, Petroski EL. Excesso de peso em adolescentes: prevalência e fatores associados. Rev Bras Ativ Fis Saúde. 2012;12(3):45-53.

13. Campos LA, Leite AJM, Almeida PC. Prevalência de sobrepeso e obesidade em adolescentes escolares do município de Fortaleza, Brasil. Rev Bras Saúde Matern Infant. 2007;7:183-90.

14. Suñé FR, Dias-da-Costa JS, Olinto MTA, Pattussi MP. Prevalência e fatores associados para sobrepeso e obesidade em escolares de uma cidade do Sul do Brasil. Cad Saúde Pública. 2007;23:1361-71.

15. Terres NG, Pinheiro RT, Horta BL, Pinheiro KAT, Horta LL. Prevalência e fatores associados ao sobrepeso e à obesidade em adolescentes. Rev Saúde Pública. 2006; 40:627-33.

16. Silva DAS, Pelegrini A, Silva AF, Grigollo LR, Petroski EL. Obesidade abdominal e fatores associados em adolescentes: comparação de duas regiôes brasileiras diferentes economicamente. Arq Bras Endocrinol Metab. 2012;56(5):291-99.

17. Programa das Naçóes Unidas para o Desenvolvimento. Atlas de Desenvolvimento Humano do Brasil. Brasília, DF: PNUD; 2013 [citado em 2018 jul 10]. Disponível em: https://goo.gl/KyyWif

18. Luiz RR, Magnanini MMF. O tamanho da amostra em investigaçôes epidemiológicas. Cad Saúde Colet. 2000;8(2):9-28.

19. Cole TJ, Lobstein T. Extended international (IOTF) body mass index cut-offs for thinness, overweight and obesity. Pediatr Obes. 2012;7(4):284-94.

20. Farias Júnior JC, Silva KS. Sobrepeso/obesidade em adolescentes escolares da cidade de João Pessoa - PB: prevalência e associação com fatores demográficos e socioeconômicos. Rev Bras Med Esporte. 2008;14(1):104-8.

21. Assumpçáo D, Barros MBA, Fisberg RM, Carandina L, Goldbaum M, Cesar CLG. Qualidade da dieta de adolescentes: estudo de base populacional em Campinas, SP. Rev Bras Epidemiol. 2012;15(3):605-16.

22. Fogelholm M, Stigman S, Huisman T, Metsamuuronen J. Physical fitness in adolescents with normal weight and overweight. Scand J Med Sci Sports. 2008;18(2):162-70.

23. Lange M, Butschalowsky HG, Jentsch F, Kuhnert R, Schaffrath RosarioA, SchlaudM, etal. ThefirstKiGGS follow-up (KiGGS Wave 1): study conduct, sample design, and response. Bundesgesundheitsblatt Gesundheitsforschung Gesundheitsschutz. 2014;57(7):747-61.

24. Singh GK, Kogan MD, van Dyck PC. Changes in state-specific childhood obesity and overweight prevalence in the United States from 2003 to 2007. Arch Pediatr Adolesc Med. 2010;164(7):598-607.

25. Instituto Brasileiro de Geografia e Estatística. Pesquisa de Orçamentos Familiares 2002-2003: antropometria e estado nutricional de crianças, adolescentes e adultos do Brasil. Rio 
de Janeiro: Ministério da Saúde, IBGE; 2006.

26. Instituto Brasileiro de Geografia e Estatística. Pesquisa Nacional de Saúde do Escolar: 2015. Rio de Janeiro: IBGE; 2016.

27. Bibiloni MD, Pons A, Tur JÁ. Prevalence of overweight and obesity in adolescents: a systematic review. ISRN Obes. 2013:392747.

28. Lampard AM, Maclehose RF, Eisenberg ME, Larson NI, Davison KK, Neumark-Sztainer D. Adolescents who engage exclusively in healthy weight control behaviors: who are they? Int J Behav Nutr Phys Act. 2016;13:5.

29. Okorodudu DO, Jumean MF, Montori VM, Romero-Corral A, Somers VK, Erwin PJ, et al. Diagnostic performance of body mass index to identify obesity as defined by body adiposity: a systematic review and meta-analysis. Int J Obes. 2010;34(5):791-99.

30. Pinto ICS, Arruda IKG, Diniz AS, Cavalcanti AMTS. Prevalência de excesso de peso e obesidade abdominal, segundo parâmetros antropométricos, e associaçáo com maturação sexual em adolescentes escolares. Cad Saúde Pública. 2010; 26:1727-37

31. Tassitano RF, Barros MVG, Tenório MCM, Bezerra J, Hallal PC. Prevalência e fatores associados ao sobrepeso e à obesidade em adolescentes, estudantes de escolas de Ensino Médio de Pernambuco, Brasil. Cad Saúde Pública. 2009; 25(12):2639-52

\section{Como citar este artigo:}

Pinto AA, Barbosa RMSP, Nahas MV, Pelegrini A. Prevalência de excesso de peso e fatores demográficos e econômicos associados em adolescentes de Manaus, a maior cidade do norte do Brasil. Rev. Aten. Saúde. 2018;16(55):64-71. 